\title{
Dependence on crossing angle of electron beam slicing in storage rings
}

\author{
A. He, F. Willeke, and L. H. Yu \\ Photon Sciences Division, Brookhaven National Laboratory, Upton, New York 11973, USA
}

(Received 3 June 2014; published 17 December 2014)

\begin{abstract}
When a low energy electron bunch $(\sim 20 \mathrm{MeV})$ crosses from top of a high energy bunch (e.g., $3 \mathrm{GeV})$ at an angle (e.g., $45^{\circ}$ ), the Coulomb force exerted on the high energy bunch by the low energy bunch will kick a very short ( $150 \mathrm{fs})$ slice from the core of the high energy bunch. The slice of electron bunch can generate ultrashort x-ray pulse. In this paper, we will give analytical expressions about the angular kicks dependence on the crossing angle by assuming a Gaussian distribution for the low energy bunch. Applying the analytical results to the storage ring bunch in NSLS-II [1], we will discuss the optimized parameters of the electron beam slicing system in order to obtain a very short slice bunch and a sufficient separation between the slice and the core bunch.
\end{abstract}

DOI: 10.1103/PhysRevSTAB.17.120704

PACS numbers: 52.59.Px

\section{INTRODUCTION}

With the rapid increase in the requirements for subpic-second $\mathrm{x}$-ray pulses in science, several approaches to generate ultrashort $\mathrm{x}$-ray pulses have been proposed. Each of them has their own characteristics, for example, the pulse duration from laser slicing [2-7] can reach the order of $100 \mathrm{fs}$, the pic-second $\mathrm{x}$-ray pulses generated by the crab cavity method [8-10] have high average flux $\left(\sim 10^{14}\right.$ photons/ sec $\left./ 0.1 \% \mathrm{bw}\right)$ and high repetition rate (several hundreds $\mathrm{MHz}$ ), the pulses produced from $\mathrm{x}$-ray free electron laser [11] are good at higher pulse energy and shorter pulse width. The electron beam slicing method $[12,13]$ is a new method to produce ultra-short X-ray pulse which has several features in space-saving, short pulse duration ( $\sim 150 \mathrm{fs})$, high flux $\left(\sim 10^{9}\right.$ photons/ sec / $0.1 \% \mathrm{bw})$, high repetition rate $(100 \mathrm{kHz}-1 \mathrm{MHz})$ and stability etc. The electron beam slicing method, as illustrated in Fig. 1, uses a focused short low energy $(\sim 20 \mathrm{MeV})$ electron bunch to slice a short electron bunch from the electron bunches in a synchrotron radiation storage ring. When the low energy electron bunch crosses from top of the high energy electron bunch at right angle, its Coulomb force will kick a short slice of high energy electrons away from the core of the storage ring electron bunch. The separated slice when passing through an undulator will radiate ultrashort $\mathrm{x}$-ray pulses at about 150 fs.

In this paper we derive the analytical expressions for the angular kicks dependence on the crossing angle induced by the Coulomb force during the crossing time of the two bunches. We discuss the parameter requirements of the

Published by the American Physical Society under the terms of the Creative Commons Attribution 3.0 License. Further distribution of this work must maintain attribution to the author(s) and the published article's title, journal citation, and DOI. electron beam slicing system, i.e., the low energy bunch beam size and the crossing angle, in order to carry out electron beam slicing experiment in the storage ring of NSLS-II. We organize this paper as follows: In Sec. II, we derive the vertical integrated angular kick as a function of the crossing angle and the 3 -D position of the storage ring bunch which includes a profile function with three scaling parameters. In Sec. III, the main performances of the e-beam slicing method is illustrated through an example. The conditions of the maximum kick and the estimated slice bunch width are given. The influence of the low energy bunch's 3-D beam size and the crossing angle on the maximum kick angle are then discussed in depth. The dependence of the slice bunch width on the crossing angle is also considered. In Sec. IV, we discuss the horizontal kick and the longitudinal energy modulation. In Sec. V, the reaction of the high energy storage ring bunch on the low energy linac bunch is described. Concluding remarks are given in Sec. VI.

\section{ANGULAR KICK $\theta_{y}$}

For simplicity, we label the high energy storage ring bunch as bunch 1 and the low energy linac bunch as bunch 2. We start from the Coulomb force exerted on the storage ring electron 1 by the low energy electron 2 , then derive the vertical angular kick $\Delta \theta_{y}$ generated by a point charge in the low energy bunch 2 . We assume the low energy bunch 2 has a Gaussian distribution in $x, y, z$ direction respectively and obtain the angular kick $\theta_{y}$ generated by the whole bunch 2 through integrating $\Delta \theta_{y}$ over the 3-D electron beam distribution of bunch 2 .

\section{A. Force exerted on the high energy storage ring electron by the low energy linac electron}

In Fig. 1, the high energy storage ring electron 1 moves in the $x o z$ plane along the direction which has angle $\varphi$ with 


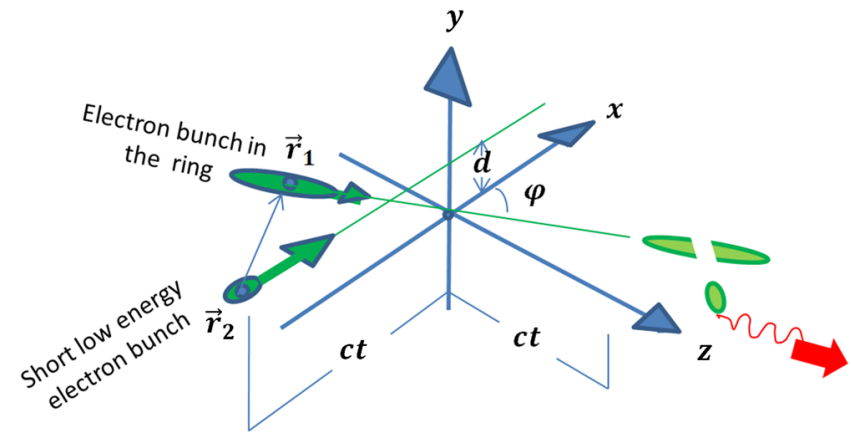

FIG. 1. Illustration of electron beam slicing.

axis " $+x$ " with relative velocity $\beta_{1}$. The low energy linac electron 2 moves in the xoy plane along the " $+x$ " direction with relative velocity $\beta_{2}$. The electric field at position $\vec{r}_{1}$ contributed by the electron 2 at position $\vec{r}_{2}$ can be expressed by [14]

$$
\vec{E}=\frac{e}{4 \pi \varepsilon_{0}} \frac{\gamma_{2}}{S^{3}} \vec{r}
$$

where $\varepsilon_{0}$ is the permittivity of free space, $S=\sqrt{\gamma_{2}^{2} x^{2}+y^{2}+z^{2}}, \quad \vec{r}=\vec{r}_{1}-\vec{r}_{2}$. The corresponding magnetic field can be calculated by $c \vec{B}=\vec{\beta}_{2} \times \vec{E}$. Then the force exerted on electron 1 by electron 2 is

$$
\begin{aligned}
\vec{F} & =e \vec{E}+e \vec{v}_{1} \times \vec{B} \\
& =e\left(1-\beta_{1} \beta_{2} \cos \varphi\right) \vec{E}+e \beta_{1} \beta_{2}\left(\cos \varphi E_{x}+\sin \varphi E_{z}\right) \vec{x},
\end{aligned}
$$

where we use $\hat{x} \times(\hat{x} \times \vec{E})=E_{x} \hat{x}-\vec{E}, \hat{z} \times(\hat{x} \times \vec{E})=E_{z} \vec{x}$.

We assume that when $t=0$ electron 1 locates at position $\left(x_{1}, y_{1}, z_{1}\right)$ and electron 2 locates at position $\left(x_{2}, y_{2}+d, z_{2}\right)$, where $d$ is the vertical distance between the high energy storage ring beam line and the low energy linac beam line. Because $v_{\vec{r}_{1}} \approx c, v_{\vec{r}_{2}} \approx c$, at time $t$, electron 1 moves to $\left(x_{1}+c t \cos \varphi, y_{1}, z_{1}+c t \sin \varphi\right)$ and electron 2 moves to $\left(x_{2}+c t, y_{2}+d, z_{2}\right)$. Inserting Eq. (1) into Eq. (2), the force can be rewritten as

$$
\vec{F}=\frac{e^{2}}{4 \pi \varepsilon_{0}} \frac{\gamma_{2}}{S^{3}}\left\{\begin{array}{l}
{\left[x+\left(\beta_{1} \beta_{2} \sin \varphi\right) z\right] \vec{x}} \\
{\left[\left(1-\beta_{1} \beta_{2} \cos \varphi\right) y\right] \vec{y} .} \\
{\left[\left(1-\beta_{1} \beta_{2} \cos \varphi\right) z\right] \vec{z}}
\end{array}\right.
$$

Using the coordinates $x_{r}, y_{r}, z_{r}$ of the storage ring bunch, the forces exerted on the storage ring electron by the linac electron in the horizontal, vertical and longitudinal direction are $F_{x_{r}}=\left(F_{x} \sin \varphi-F_{z} \cos \varphi\right), \quad F_{y_{r}}=F_{y}$, $F_{z_{r}}=\left(F_{x} \cos \varphi+F_{z} \sin \varphi\right)$, respectively. Therefore the force, expressed in terms of the coordinates of the storage ring bunch, is

$$
\left(\begin{array}{c}
F_{x_{r}} \\
F_{y_{r}} \\
F_{z_{r}}
\end{array}\right)=\frac{e^{2}}{4 \pi \varepsilon_{0}} \frac{\gamma_{2}}{S^{3}}\left(\begin{array}{c}
x \sin \varphi+z\left(\beta_{1} \beta_{2}-\cos \varphi\right) \\
y\left(1-\beta_{1} \beta_{2} \cos \varphi\right) \\
(x \cos \varphi+z \sin \varphi)
\end{array}\right),
$$

where $x=-\left(x_{2}-x_{1}\right)-\operatorname{ct}(1-\cos \varphi), y=-\left(y_{2}-y_{1}\right)-d$, $z=-\left(z_{2}-z_{1}\right)+c t \sin \varphi$. Notice the force components in the storage ring coordinate system is expressed in terms of the low energy bunch coordinates.

\section{B. Angular kick $\Delta \theta_{y}$ by single low energy particle}

We assume each low energy particle's point charge is $q_{2}$. According to Eq. (4), the kick force on electron 1 in the vertical direction $\vec{y}$ can be expressed as

$$
F_{y}=-\frac{e q_{2}}{4 \pi \varepsilon_{0}} \frac{\gamma_{2}}{S^{3}}\left(1-\beta_{1} \beta_{2} \cos \varphi\right)\left(d+y_{2}-y_{1}\right) .
$$

The relation between the kick force and the kick angle can be expressed as

$$
F_{y}=\gamma_{1} m c \frac{d \theta_{y}}{d t}=\frac{E_{1}}{c} \frac{d \theta_{y}}{d t},
$$

where we use $\frac{d y}{d t} \approx c \theta_{y}$ due to $v_{\vec{r}_{1}} \approx c, E_{1}$ is the energy of the storage ring electron 1 . The angular kick $\Delta \theta_{y}$ generated by a point charge in the low energy bunch 2 can be obtained by integrating $d \theta_{y}=\left(c F_{y} / E_{1}\right) d t$ over the crossing time:

$$
\begin{aligned}
\Delta \theta_{y} & =\frac{e q_{2} \gamma_{2} c}{4 \pi \varepsilon_{0} E_{1}} \int_{-\infty}^{+\infty} \frac{d t}{S^{3}}\left(1-\beta_{1} \beta_{2} \cos \varphi\right)\left(d+y_{2}-y_{1}\right) \\
& =\frac{e q_{2} Z_{0} c}{2 \pi E_{1}} \cdot \frac{\gamma_{2}\left(1-\beta_{1} \beta_{2} \cos \varphi\right)}{\left[\gamma_{2}^{2}(1-\cos \varphi)^{2}+\sin ^{2} \varphi\right]^{1 / 2}} \cdot \frac{d+y_{2}-y_{1}}{a^{2}},
\end{aligned}
$$

where $Z_{0}=\frac{1}{\varepsilon_{0} c}=377 \Omega$ and

$$
\begin{aligned}
a^{2}= & \frac{\gamma_{2}^{2}}{\gamma_{2}^{2}(1-\cos \varphi)^{2}+\sin \varphi^{2}} \\
& \times\left[\left(x_{2}-x_{1}\right) \sin \varphi+\left(z_{2}-z_{1}\right)(1-\cos \varphi)\right]^{2} \\
& +\left(d+y_{2}-y_{1}\right)^{2} .
\end{aligned}
$$

In Eq. (7), we removed a minus sign for the $\Delta \theta_{y}$ which is due to the minus sign of Eq. (5). That means we define $\Delta \theta_{y}$ positive if the electron is kicked downward as shown in Fig. 1.

\section{Angular kick $\boldsymbol{\theta}_{\boldsymbol{y}}$ by a Gaussian low energy bunch}

We assume the low energy bunch 2 has a Gaussian distribution in $x, y, z$ direction and the RMS bunch size is $\sigma_{x}, \sigma_{y}, \sigma_{z}$, respectively. Integrating $\Delta \theta_{y}$ over the whole low energy bunch 2 , we obtain the angular kick generated by the low energy bunch on the storage ring electron as: 
$\theta_{y}=\frac{e q_{2} Z_{0} c}{2 \pi E_{1}} \cdot \frac{\gamma_{2}\left(1-\beta_{1} \beta_{2} \cos \varphi\right)}{\left[\gamma_{2}^{2}(1-\cos \varphi)^{2}+\sin ^{2} \varphi\right]^{1 / 2}} \cdot \frac{1}{(2 \pi)^{3 / 2} \sigma_{x} \sigma_{y} \sigma_{z}} \cdot I_{y}$,

where

$$
I_{y}=\iiint_{V} e^{-\frac{x_{2}^{2}}{2 \sigma_{x}^{2}}} e^{-\frac{y_{2}^{2}}{2 \sigma_{y}^{2}}} e^{-\frac{z_{2}^{2}}{2 \sigma_{z}^{2}}} \cdot \frac{d+y_{2}-y_{1}}{a^{2}} d x_{2} d y_{2} d z_{2} .
$$

Using the variable transform $x_{2}=\sqrt{2} \sigma_{x} x, y_{2}=\sqrt{2} \sigma_{y} y$, $z_{2}=\sqrt{2} \sigma_{z} z$, and the following defined scaling parameters:

$$
\begin{aligned}
\rho & \equiv \sqrt{\frac{\gamma_{2}^{2}}{\gamma_{2}^{2}(1-\cos \varphi)^{2}+\sin ^{2} \varphi} \cdot \frac{\sigma_{x}^{2} \sin ^{2} \varphi+\sigma_{z}^{2}(1-\cos \varphi)^{2}}{\sigma_{y}^{2}}} \\
\bar{y}_{1} & \equiv \frac{d-y_{1}}{\sqrt{2} \sigma_{y}} \\
\bar{u}_{1} & \equiv \frac{x_{1} \sin \varphi+z_{1}(1-\cos \varphi)}{\sqrt{2 \sigma_{x}^{2} \sin ^{2} \varphi+2 \sigma_{z}^{2}(1-\cos \varphi)^{2}}},
\end{aligned}
$$

the triple integral of Eq. (9) can be reduced to the double integral:

$$
I_{y}=2 \sqrt{\pi} \sigma_{x} \sigma_{z} \iint e^{-y^{2}-u^{2}} \frac{y+\bar{y}_{1}}{b^{2}} d y d u,
$$

where $b^{2}=\left(y+\bar{y}_{1}\right)^{2}+\rho^{2}\left(u-\bar{u}_{1}\right)^{2}$. Using the integral equation $\frac{\beta}{\pi} \int \frac{e^{-x^{2}}}{(x-\alpha)^{2}+\beta^{2}} d x=\operatorname{Re}[W(\alpha+i|\beta|) \operatorname{Sign}(\beta)]$ with $W(u)=e^{-u^{2}} \operatorname{erfc}(-i u)$ and error function $\operatorname{erfc}(x)=$ $\frac{2}{\sqrt{\pi}} \int_{x}^{+\infty} e^{-x^{2}} d x$, Eq. (11) can be written as

$$
\begin{aligned}
I_{y}= & 2 \pi^{3 / 2} \sigma_{x} \sigma_{z} \int_{0}^{\infty} \operatorname{Re}\left[W\left(\bar{u}_{1}+i y\right)\right] \\
& \times\left[e^{-\left(\rho y-\bar{y}_{1}\right)^{2}}-e^{-\left(\rho y+\bar{y}_{1}\right)^{2}}\right] d y .
\end{aligned}
$$

We now transform the storage ring particle's coordinates from the coordinate system defined relative to low energy bunch shown in Fig. 1 to the storage ring coordinate $x_{r}, y_{r}, z_{r}$ by using the variable transform $x_{1}=z_{r} \cos \varphi+x_{r} \sin \varphi, y_{1}=y_{r}, z_{1}=z_{r} \sin \varphi-x_{r} \cos \varphi$, then $\bar{u}_{1}$ in Eq. (10) becomes $\bar{u}_{1} \equiv \frac{z_{r} \sin \varphi+x_{r}(1-\cos \varphi)}{\sqrt{2 \sigma_{x}^{2} \sin ^{2} \varphi+2 \sigma_{z}^{2}(1-\cos \varphi)^{2}}}$. Inserting Eq. (12) into Eq. (8) and using the previous coordinates transform from $x_{1}, y_{1}, z_{1}$ to $x_{r}, y_{r}, z_{r}$, we obtain the final angular kick $\theta_{y}$ as:

$\theta_{y}=\frac{e q_{2} Z_{0} c}{2 \pi E_{1}} \frac{\gamma_{2}\left(1-\beta_{1} \beta_{2} \cos \varphi\right)}{\sqrt{\gamma_{2}^{2}(1-\cos \varphi)^{2}+\sin ^{2} \varphi}} \frac{1}{\sqrt{2} \sigma_{y}} f_{y}\left(\rho, \bar{u}_{1}, \bar{y}_{1}\right)$,

where $f_{y}$ gives the profile as a function of the high energy electron's position

$$
\begin{aligned}
f_{y}\left(\rho, \bar{u}_{1}, \bar{y}_{1}\right)= & \int_{0}^{\infty} \operatorname{Re}\left[W\left(\bar{u}_{1}+i y\right)\right] \\
& \times\left[e^{-\left(\rho y-\bar{y}_{1}\right)^{2}}-e^{-\left(\rho y+\bar{y}_{1}\right)^{2}}\right] d y .
\end{aligned}
$$

$\rho, \bar{u}_{1}, \bar{y}_{1}$ are given by

$$
\begin{aligned}
\rho & \equiv \sqrt{\frac{\gamma_{2}^{2}}{\gamma_{2}^{2}(1-\cos \varphi)^{2}+\sin ^{2} \varphi} \cdot \frac{\sigma_{x}^{2} \sin ^{2} \varphi+\sigma_{z}^{2}(1-\cos \varphi)^{2}}{\sigma_{y}^{2}}} \\
\bar{y}_{1} & \equiv \frac{d-y_{r}}{\sqrt{2} \sigma_{y}} \\
\bar{u}_{1} & \equiv \frac{z_{r} \sin \varphi+x_{r}(1-\cos \varphi)}{\sqrt{2 \sigma_{x}^{2} \sin ^{2} \varphi+2 \sigma_{z}^{2}(1-\cos \varphi)^{2}}}
\end{aligned}
$$

Equation (13) gives the angular kick as a function of the electron's 3-D position $x_{r}, y_{r}, z_{r}$ in the storage ring bunch 1 . The profile function Eq. (14) describes the profile of the slice bunch and can be used to estimate the pulse width of the slice.

\section{NUMERICAL ANALYSIS}

For this slicing method, we require that the vertical angular deviation of the slice bunch is large enough for the slice to be separated from the core bunch and the pulse length of the slice is small enough to radiate femtosecond $\mathrm{x}$-ray. For the preliminary design, according to the kick function Eq. (13), we can determine the requirements on the low energy bunch performances, pick the interaction position in the storage ring of NSLS-II and choose the crossing angle between the two bunches.

The value of the kick angle in Eq. (13) can be separated into two parts: the nominal kick angle

$$
\theta_{y 0}=\frac{e q_{2} Z_{0} c}{2 \pi E_{1}} \frac{\gamma_{2}\left(1-\beta_{1} \beta_{2} \cos \varphi\right)}{\sqrt{\gamma_{2}^{2}(1-\cos \varphi)^{2}+\sin ^{2} \varphi}} \frac{1}{\sqrt{2} \sigma_{y}}
$$

and the coefficient $f_{y}\left(\rho, \bar{u}_{1}, \bar{y}_{1}\right)$ which determines the profile of the slice bunch. Equation (16) shows that the nominal kick angle is proportional to the low energy bunch charge, inversely proportional to the storage ring energy $E_{1}$ and the vertical beam size $\sigma_{y}$ of the low energy bunch. It also depends on the crossing angle $\varphi$, but is not sensitive to the low energy bunch energy $\gamma_{2}$ when it is much larger than one. From the cost point of view, we prefer the energy $\gamma_{2}$ of bunch 2 as low as possible. Therefore in our discussion, we choose $E_{2}=20 \mathrm{MeV}$ corresponding to $\gamma_{2} \approx 39$. The profile function $f_{y}\left(\rho, \bar{u}_{1}, \bar{y}_{1}\right)$ is basically a function of the particle position $x_{r}, y_{r}, z_{r}$ of high energy electrons in the storage ring coordinate system and the crossing angle $\varphi$ of the two bunches through the scaling function Eq. (15). The profile function gives the maximum angular kick condition and also describes the slice pulse width. 
In the following analysis, we first give an example to explain what kind of performances of the e-beam slicing method we can expect. Then we will discuss how the low energy bunch size $\sigma_{x}, \sigma_{y}, \sigma_{z}$ and the crossing angle $\varphi$ influence the maximum kick angle $\theta_{y, \max }$ and the slice width.

\section{A. Numerical example}

To estimate the possible range of the performances for the e-beam slicing experiment, we give a numerical example at NSLS-II [1] for the crossing angle $\varphi=90^{\circ}$. We assume the kick point is at a position in the storage ring where $\beta_{x}=3.8 \mathrm{~m}, \beta_{y}=25 \mathrm{~m}$, if the horizontal and vertical emittance is $\varepsilon_{x}=1 \mathrm{~nm}, \varepsilon_{y}=10 \mathrm{pm}$, respectively, then the horizontal and vertical RMS beam divergence is $\sigma_{x}^{\prime}=16 \mu \mathrm{rad}, \sigma_{y}^{\prime}=0.6 \mu \mathrm{rad}$, respectively.

To separate the slice from the core bunch of the storage ring we estimate that the vertical angular kick should be more than 5 times larger than $\sigma_{y}{ }^{\prime}$, i.e., $\theta_{y}>3 \mu \mathrm{rad}$. We assume the $20 \mathrm{MeV}$ low energy electron bunch with charge of $q_{2}=200 \mathrm{pC}$ is focused to the horizontal and vertical beam size of $\sigma_{z}=\sigma_{y}=35 \mu \mathrm{m}$, and compressed to the longitudinal beam size $\sigma_{x}=35 \mu \mathrm{m}$, i.e., bunch length of about $120 \mathrm{fs}$. As an example, taking the storage ring energy as $E_{1}=3 \mathrm{GeV}$, for crossing angle $\varphi=90^{\circ}$, we find the nominal kick angle as $\theta_{y 0}\left(90^{\circ}\right)=$ $\frac{e q_{2} Z_{0} c}{2 \pi E_{1}} \frac{\gamma_{2}}{\sqrt{\gamma_{2}^{2}+1}} \frac{1}{\sqrt{2} \sigma_{y}}=24 \mu \mathrm{rad}$. The profile parameter $\rho=\sqrt{\frac{\gamma_{2}^{2}}{\gamma_{2}^{2}+1} \cdot \frac{\sigma_{x}^{2}+\sigma_{z}^{2}}{\sigma_{y}^{2}}}=1.4$. We choose the distance between the storage ring bunch's beam-line and the low energy bunch's beam-line as $d=\sqrt{2} \sigma_{y}=50 \mu \mathrm{m}$, which we find to give maximum kick. Assume $x_{r}=y_{r}=z_{r}=0$, i.e., when the electron in the storage ring bunch arrives at the origin of the coordinate system, the center of the low energy bunch just arrives at the position on the top of the origin by vertical distance $d=50 \mu \mathrm{m}$, then we have $\bar{y}_{1}=1$, $\bar{u}_{1}=0$, and we find $f_{y}=0.54$. The kick angle is given by $\theta_{y}\left(90^{\circ}\right)=\theta_{y 0}\left(90^{\circ}\right) f_{y}=13 \mu \mathrm{rad}$, much larger than the required $3 \mu \mathrm{rad}$ for the separation from the core.

The width of the slice is estimated by the width in $\bar{u}_{1}$ of the profile $f_{y}$ at $\bar{y}_{1}=1$ and the horizontal beam size of the storage ring bunch adding in quadrature (we will discuss the slice width in more detail later in Sec. III D). For the above example $\sigma_{x}=\sigma_{y}=\sigma_{z}=35 \mu \mathrm{m}$, at the kick point with $\beta_{x}=3.8 \mathrm{~m}$ and $\varepsilon_{x}=1 \mathrm{~nm}$, the horizontal FWHM of the storage ring bunch is about $145 \mu \mathrm{m}$ and at $\rho=1.4$ the FWHM of $\bar{u}_{1}$ is 2.5 . Then the estimated FWHM of the slice bunch is about $227 \mu \mathrm{m}$ which corresponds to $760 \mathrm{fs}$. As an estimate, we use an aperture to select $\mathrm{x}$-rays from those electrons in the slice whose vertical angular divergence is larger than half of the maximum kick angle, then the peak to peak pulse width of the slice is $760 \mathrm{fs}$. Thus the estimated RMS value of slice width should be estimated roughly as one fourth of this peak to peak value, i.e., 190 fs. We can see the contribution of the horizontal crossing time dominates the pulse width. But, as we will discuss in more detail later in Sec. III D, we find that when the low energy bunch crosses the high energy bunch with an angle of $45^{\circ}$ rather than $90^{\circ}$, this problem of horizontal crossing time can be overcome. Then, again, the width of $\bar{u}_{1}$ becomes important, and we can reach the slice length of the order of $107 \mathrm{fs}$.

\section{B. Profile function $f_{y}$}

To understand the relation of the angular kick and the scaling parameters, we plot the function $f_{y}$ in Fig. 2 for the case of $\rho=1$.4. The plot shows the maximum point locates at $\bar{u}_{1}=0, \bar{y}_{1}=1$. A set of plots of $f_{y}$ as a function of $\bar{y}_{1}$ at $\bar{u}_{1}=0$ for different $\rho$ are shown in Fig. 3. The maximum is always approximately at $\bar{y}_{1}=1$. That means if we choose the vertical distance between the two bunches center is $d=\sqrt{2} \sigma_{y}$, the electron in the storage ring bunch located at $x_{r}=0, y_{r}=0, z_{r}=0$ will receive the maximum kick $\theta_{y, \max }$. The value of the maximum kick can be adjusted through the scaling parameter $\rho$ by changing the crossing angle $\varphi$ and the low energy bunch size $\sigma_{x}, \sigma_{y}, \sigma_{z}$. We also plot the profile of $f_{y}$ as a function of $\bar{u}_{1}$ for $\bar{y}_{1}=1$ in Fig. 4 for different $\rho$. The width in $\bar{u}_{1}$ of the profile $f_{y}$ at $\bar{y}_{1}=1$ can be used to estimate the width of the slice.

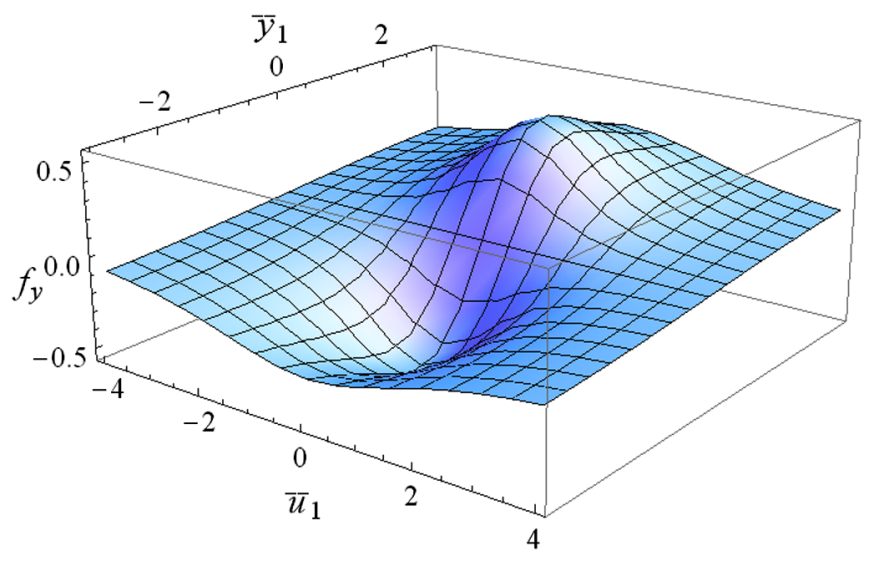

FIG. 2. Profile function $f_{y}$.

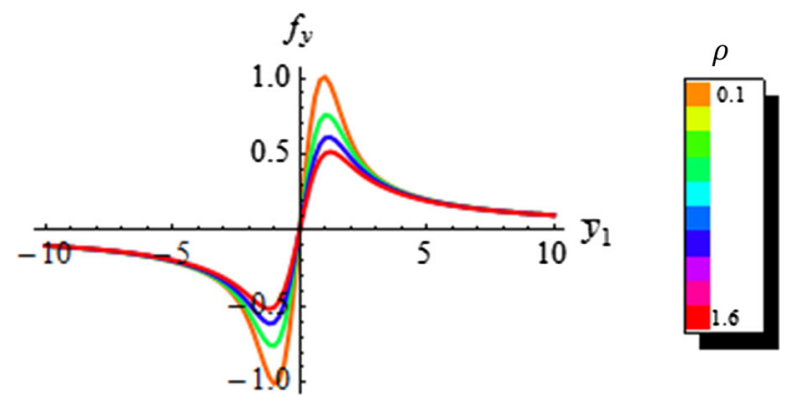

FIG. 3. Profile on axis $\bar{u}_{1}=0$. The maximum $f_{y}$ locates at $\bar{y}_{1}=1$. 


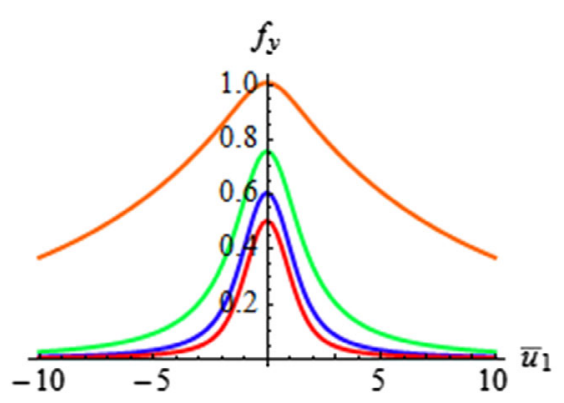

FIG. 4. Profile on axis $\bar{y}_{1}=1$. Show that decreasing $\rho$ will advantage to increase the pulse width.

\section{Maximum kick value $\boldsymbol{\theta}_{\boldsymbol{y} \text {, max }}$}

According to the previous discussion, if we design the distance between the low energy bunch's beam line and the storage ring beam line $d=\sqrt{2} \sigma_{y}$ and when the two bunches' centers arrive at $x_{r}=0, z_{r}=0$ with only a vertical separation $d$ that means we have $\bar{y}_{1}=1, \bar{u}_{1}=0$, then the vertical kick reaches maximum $\theta_{y, \max }=\theta_{y}\left(x_{r}=\right.$ $\left.0, y_{r}=0, z_{r}=0\right)=\theta_{y 0} f\left(\rho, \bar{u}_{1}=0, \bar{y}_{1}=1\right)$. The maximum kick angle $\theta_{y, \max }$ is still a function of the low energy bunch's 3-D beam size $\sigma_{x}, \sigma_{y}, \sigma_{z}$ and the crossing angle $\varphi$. In this section, we will discuss the dependence of $\theta_{y, \max }$ on $\sigma_{x}, \sigma_{y}, \sigma_{z}$ and $\varphi$, and find out the relative importance of $\sigma_{x}, \sigma_{y}, \sigma_{z}$ at different crossing angle. Based on the practical performance of the low energy linac compressor, the 3-D beam size $\sigma_{x}, \sigma_{y}, \sigma_{z}$ discussed are from $25 \mu \mathrm{m}$ to $45 \mu \mathrm{m}$. The crossing angle discussed is from $0^{\circ}$ to $90^{\circ}$.

We plot the maximum kick $\theta_{y, \max }$ as a function of the crossing angle $\varphi$ for the low energy beam size $\sigma_{x}=\sigma_{y}=\sigma_{z}$ in Fig. 5. The kick angle increases with the increase of the crossing angle when $\varphi>5^{\circ}$. And the plot also shows that the smaller the low energy beam size is, the stronger the kick is. In order to make the relation between the low energy beam size and the slice kick angle more clear, we also plot the dependence of the maximum kick angle on the beam size for different crossing angles in Fig. 6.

In general, we need large kick angle, then we need small 3-D beam size $\sigma_{x}, \sigma_{y}, \sigma_{z}$ and large crossing angle $\varphi$. If we consider the relative importance of the 3-D beam size to the kick value, we will notice that in Eq. (15) the vertical beam

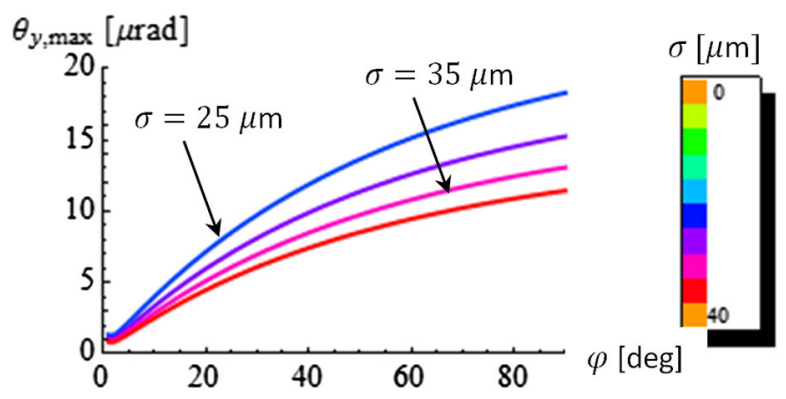

FIG. 5. The maximum kick angle $\theta_{y, \max }$ as a function of the crossing angle $\varphi$ for different beam size $\sigma$ when $\sigma=\sigma_{x}=\sigma_{y}=\sigma_{z}$.

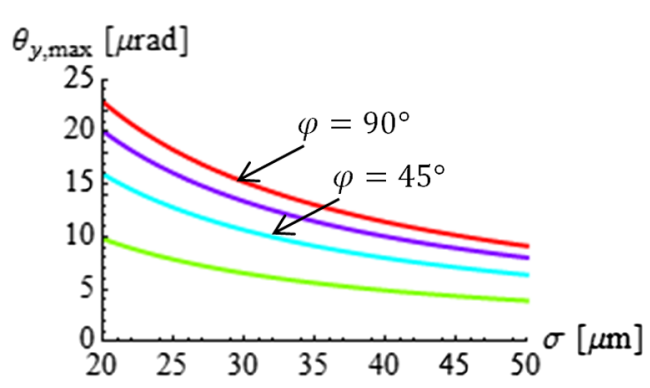

$\varphi$ [deg]

FIG. 6. The maximum kick angle $\theta_{y, \max }$ as a function of the beam size $\sigma_{x}=\sigma_{y}=\sigma_{z}$ for different crossing angle $\varphi$.

size $\sigma_{y}$ of the low energy bunch and its other two beam size $\sigma_{x}, \sigma_{z}$ locate at different positions in the scaling parameter $\rho$, and in the nominal kick function Eq. (16) only the vertical beam size $\sigma_{y}$ appears. In addition, we remark that the crossing angle $\varphi$ influences the relative importance of $\sigma_{x}$ and $\sigma_{z}$ to the scaling parameter $\rho$.

With the vertical beam size $\sigma_{y}$ fixed, we found in the range of crossing angle $0^{\circ}-90^{\circ}$, to obtain the same kick angle smaller $\sigma_{x}$ is more efficient than smaller $\sigma_{z}$. To see this, we choose $\sigma_{y}=35 \mu \mathrm{m}$ as an example, and then we plot several contour figures of the kick value as a function of the longitudinal and horizontal beam size $\sigma_{x}, \sigma_{z}$ for different crossing angle $\varphi=22.5^{\circ}, 45^{\circ}, 67.5^{\circ}, 90^{\circ}$ in Fig. 7 . The color represents the values of the kick angle. The more it is close to the yellow, the larger the kick angle is. The same black dash lines present the same kick values and the slope of the dash line indicates which beam size influences the kick angle more. The steeper the slope is, the less important $\sigma_{x}$ is while the more important $\sigma_{z}$ is to the kick. For example, the top-right plot $\left(\varphi=45^{\circ}\right)$ in Fig. 7 shows that to obtain the same kick angle it is more efficient to short the low energy bunch's longitudinal beam size $\sigma_{x}$ than to short its horizontal beam size $\sigma_{z}$. While the bottom-right plot $\left(\varphi=90^{\circ}\right)$ in Fig. 7 indicates that the longitudinal beam size $\sigma_{x}$ and the horizontal beam size $\sigma_{z}$ have the same importance to kick angle when the crossing angle is $\varphi=90^{\circ}$.

The relative importance between the vertical beam size $\sigma_{y}$ and the other two beam size $\sigma_{x}, \sigma_{z}$ is complicated. Therefore we limit our discussion in the practical range of $25 \mu \mathrm{m}-45 \mu \mathrm{m}$ for the 3-D beam size. To figure out the relative importance of $\sigma_{x}$ and $\sigma_{y}$ we do the following scanning: (i) First we fix the value of $\sigma_{z}$ and plot the contour plots of the kick angle as a function of $\sigma_{x}$ and $\sigma_{y}$ for different crossing angle $\varphi=22.5^{\circ}, 45^{\circ}, 67.5^{\circ}, 90^{\circ}$ with $\sigma_{y}$. (ii) Then we scan $\sigma_{z}$ from $25 \mu \mathrm{m}$ to $45 \mu \mathrm{m}$ with the step size $1 \mu \mathrm{m}$ to plot the corresponding contour figures as step 1. Comparing these contour plots, we find that with the increase of the crossing angle the slope of the contour line will rotate clockwise. That means the importance of $\sigma_{y}$ to the kick value is gradually increasing while the importance of $\sigma_{x}$ is decreasing with the increase of the crossing angle. 

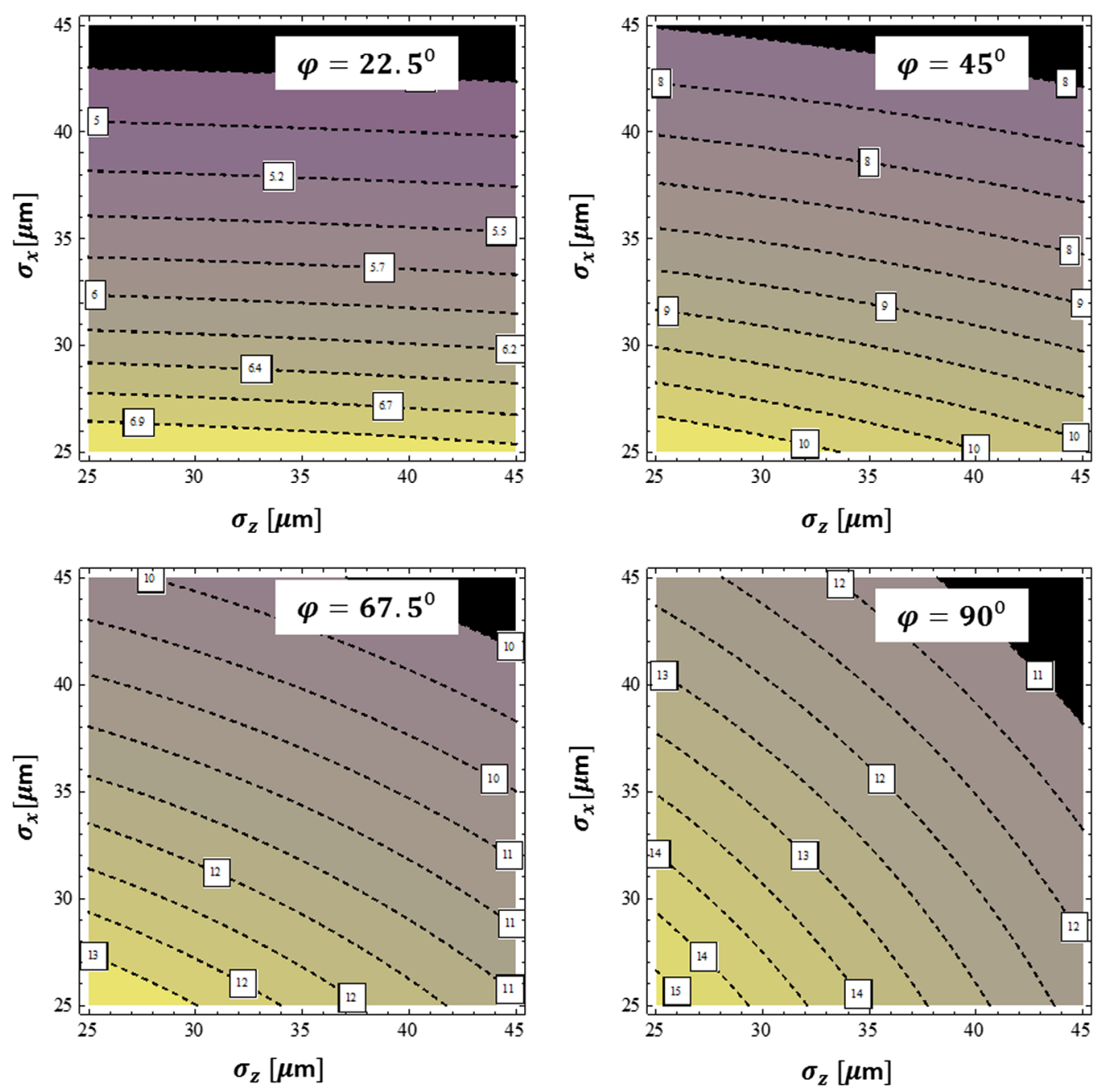

FIG. 7. Kick angle contour for $\sigma_{y}=35 \mu \mathrm{m}$. These pictures show the contour of the maximum kick angle $\theta_{y \text {,max }}$ as a function of the longitudinal and horizontal beam size $\sigma_{x}, \sigma_{z}$ for different crossing angle $\varphi=22.5^{\circ}, 45^{\circ}, 67.5^{\circ}, 90^{\circ}$. The color represents the values of the kick angle. The more it is closer to the yellow, the larger the kick angle is.

We also find the influence of the horizontal beam size $\sigma_{z}$ on the rotation and the relative slope value can be ignored in the discussed range of $25 \mu \mathrm{m}-45 \mu \mathrm{m}$ for the 3-D beam size. As an example for $\sigma_{z}=35 \mu \mathrm{m}$, the contour plots of the kick value as a function of the longitudinal and vertical beam size $\sigma_{x}, \sigma_{y}$ for different crossing angle are shown in Fig. 8. The clockwise rotation of the contour line clearly indicates that to reach a kick value, for $45^{\circ}$ crossing angle smaller $\sigma_{x}$ is more important than smaller $\sigma_{y}$ as shown in the top-left plot of Fig. 8, while for $90^{\circ}$ crossing angle to short $\sigma_{y}$ is more efficient than do $\sigma_{x}$ as shown in the bottom-left of Fig. 8. We do the same scanning process to study the relative importance of $\sigma_{y}$ and $\sigma_{z}$. The results, which are independent from the value of $\sigma_{x}$ in the discussed range of $25 \mu \mathrm{m}-45 \mu \mathrm{m}$ for the 3-D beam size, show that a smaller vertical beam size $\sigma_{y}$ is more important to the kick value than a smaller horizontal beam size $\sigma_{z}$ for the crossing angle discussed. An example plot is shown in Fig. 9.
Table I gives a conclusion about the relative importance of the 3-D beam size to the kick value $\theta_{y \text {,max }}$ for different crossing angle situations, i.e., for $45^{\circ}$ crossing angle, to increase the kick angle shortening the longitudinal beam size $\sigma_{x}$ is the most efficient way and for $90^{\circ}$ crossing angle shortening the vertical beam size $\sigma_{y}$ is more efficient than shortening $\sigma_{x}$ and $\sigma_{z}$. These conclusions are justified in the range discussed around $25 \mu \mathrm{m}-45 \mu \mathrm{m}$ of 3-D beam size. The characteristics of the low energy bunch's 3-D beam size with different weights on the kick value makes the optimization of the low energy beam size more flexible. For some examples in Table II, if we manipulate relative beam size in different directions we can obtain different kick values.

\section{Slice width}

The width of the slice beam is another important parameter. From the expression of $\bar{u}_{1}$ in Eq. (15), we have 

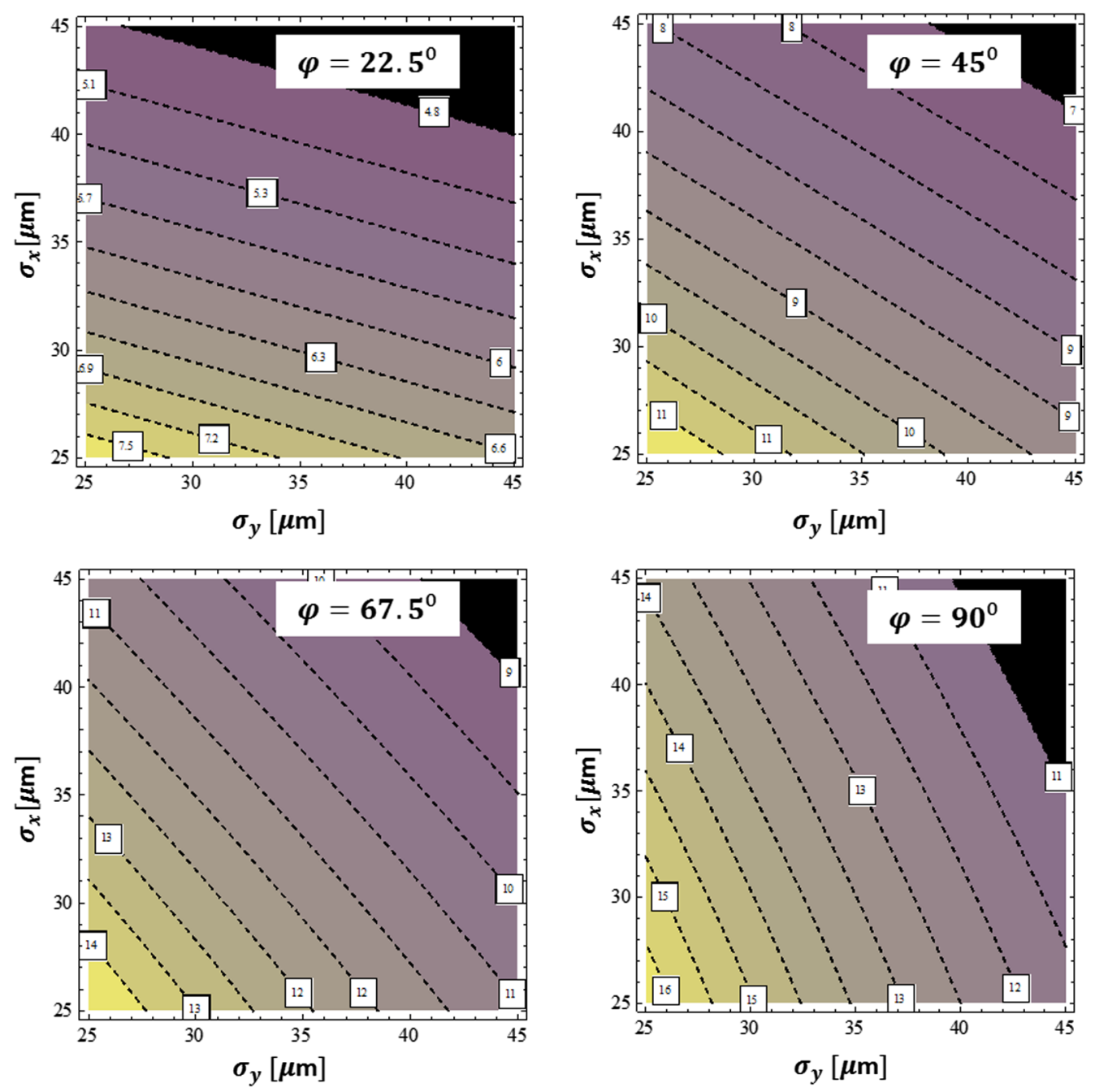

FIG. 8. Kick angle contour for $\sigma_{z}=35 \mu \mathrm{m}$. These pictures show the contour of the maximum kick angle $\theta_{y \text {,max }}$ as a function of the longitudinal and vertical beam size $\sigma_{x}, \sigma_{y}$ for different crossing angle $\varphi=22.5^{\circ}, 45^{\circ}, 67.5^{\circ}, 90^{\circ}$. The color represents the values of the kick angle. The more it is closer to the yellow, the larger the kick angle is.

$$
z_{r}=\bar{u}_{1} \sqrt{2 \sigma_{x}^{2}+2 \sigma_{z}^{2}\left(\frac{1-\cos \varphi}{\sin \varphi}\right)^{2}}+\left(\frac{\cos \varphi-1}{\sin \varphi}\right) x_{r}
$$

This equation shows the slice width is affected by two independent terms: one term, being proportional to $\bar{u}_{1}$, due to the vertical kick from the linac bunch and the other term, being proportional to $x_{r}$, due to the horizontal crossing time of the low energy bunch when it passes across the high energy bunch from top. These terms add in quadrature to provide the slice width. To estimate the slice width, we assume the horizontal distribution of the storage ring bunch is Gaussian, and we also approximate the profile of $\bar{u}_{1}$ as Gaussian. Equation (17) shows the crossing angle $\varphi$ influences the vertical kick term $\bar{u}_{1}$ and the horizontal crossing time term $x_{r}$. It is possible to reduce the pulse length due to the crossing time by reducing the crossing angle, for example from $90^{\circ}$ to $45^{\circ}$ between the forward direction of the linac beam and the storage ring beam. This is illustrated in Fig. 10 showing for $45^{\circ}$ crossing angle the linac bunch has a velocity component parallel to the storage ring bunch making the slice shorter. We display this effect using the contour plots in Fig. 11 which shows the phase space distribution with color scale representing the kick angle for the $90^{\circ}$ crossing and $45^{\circ}$ crossing, respectively. The parameters used to plot Fig. 11 are the same as the example in Sec. III A. Using the same way in Sec. III A, the estimated RMS value of bunch length decreases from $190 \mathrm{fs}$ for $90^{\circ}$ crossing angle to $107 \mathrm{fs}$ for $45^{\circ}$ crossing angle at the expense of a reduction of the maximum vertical kick angle $\theta_{y, \max }$ from $13 \mu \mathrm{rad}$ to $9 \mu \mathrm{rad}$. More numerical examples are given in Table II. In Fig. 12 we plot an example curve about the variation of the estimated RMS slice width with the crossing angle. When the 3-D beam size of the low energy bunch keep the same value, the slice width is independent of the charge of the low energy bunch. Therefore the decrease of the kick angle because we choose 

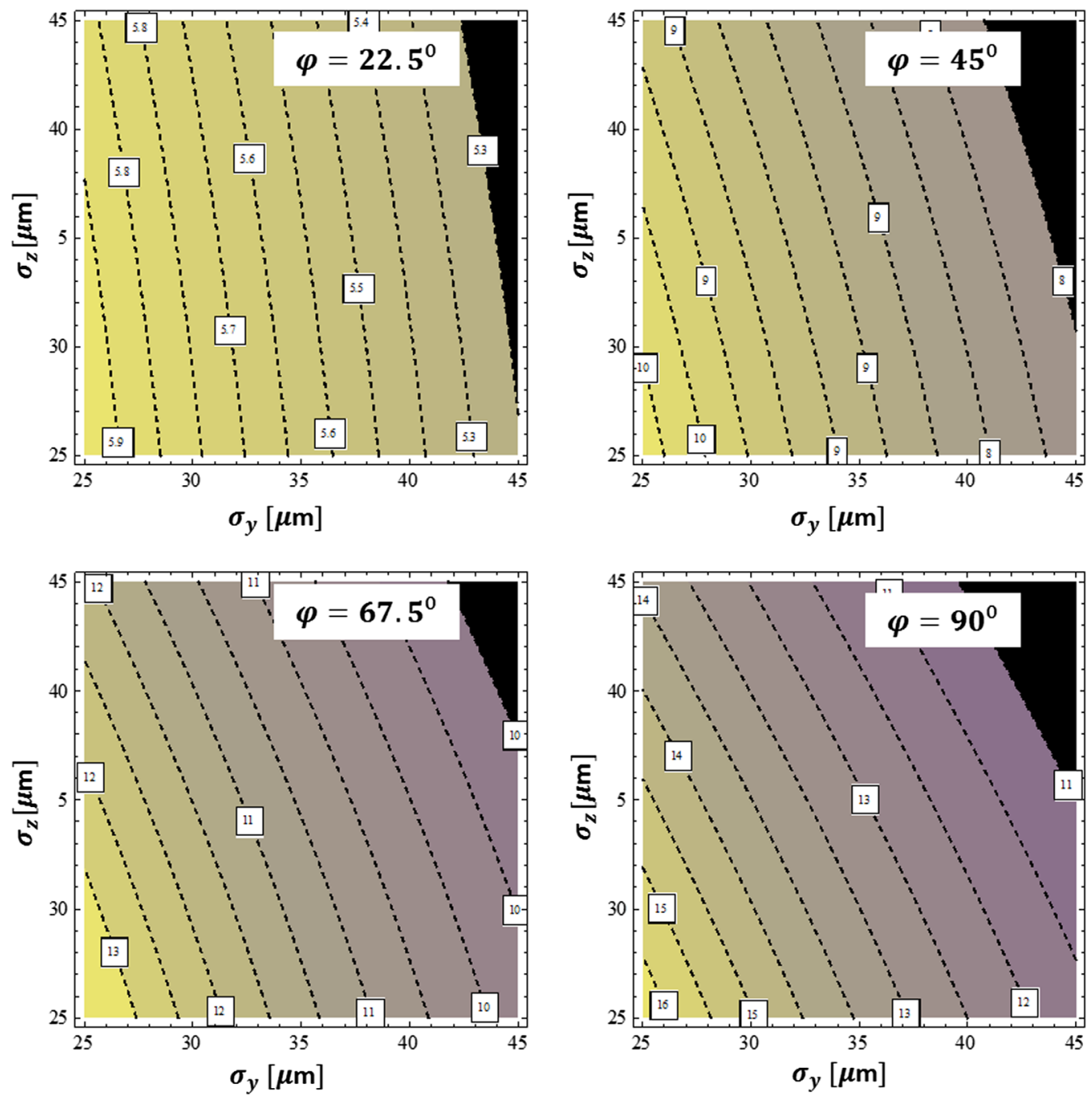

FIG. 9. Kick angle contour for $\sigma_{x}=35 \mu \mathrm{m}$. These pictures show the contour of the maximum kick angle $\theta_{y \text {,max }}$ as a function of the vertical and horizontal beam size $\sigma_{y}, \sigma_{z}$ for different crossing angle $\varphi=22.5^{\circ}, 45^{\circ}, 67.5^{\circ}, 90^{\circ}$. The color represents the values of the kick angle. The more it is closer to the yellow, the larger the kick angle is.

a smaller crossing angle to shorten the slice width can be made up by increasing the charge of low energy bunch. At the same time, due to the space charge effects, with increasing the charge of low energy bunch we also need to increase the energy of low energy bunch to keep the same 3-D beam size.

TABLE I. The relative importance of the 3-D beam size $\sigma_{x}, \sigma_{y}, \sigma_{z}$ to the kick angle for different crossing angle, i.e., $\sigma_{y}<$ $\sigma_{x}=\sigma_{z}$ means that to reach a kick value smaller vertical beam size $\sigma_{y}$ is more important and the longitudinal and horizontal beam size $\sigma_{x}, \sigma_{z}$ has the same weight on the kick value.

\begin{tabular}{lc}
\hline \hline crossing angle & relative importance \\
\hline $22.5^{\circ}$ & $\sigma_{x}<\sigma_{y}<\sigma_{z}$ \\
$45^{\circ}$ & $\sigma_{x}<\sigma_{y}<\sigma_{z}$ \\
$62.5^{\circ}$ & $\sigma_{y}<\sigma_{x}<\sigma_{z}$ \\
$90^{\circ}$ & $\sigma_{y}<\sigma_{x}=\sigma_{z}$ \\
\hline \hline
\end{tabular}

\section{HORIZONTAL KICK $\theta_{x r}$ AND LONGITUDINAL ENERGY MODULATION $\Delta \gamma$}

When the low energy linac bunch crossing pass through the high energy storage ring bunch, except the vertical kick $\theta_{y}$ the storage ring bunch also experiences the horizontal kick $\theta_{x r}$ and the longitudinal energy modulation $\Delta \gamma$ induced by the low energy bunch's electromagnetic field.

\section{A. Horizontal kick $\boldsymbol{\theta}_{\boldsymbol{x}}$}

According to Eq. (4), the horizontal kick force on storage ring electron is expressed as

$$
\begin{aligned}
F_{x r}= & \frac{e q_{2}}{4 \pi \varepsilon_{0}} \frac{\gamma_{2}}{S^{3}}\left[\left(x_{1}-x_{2}\right) \sin \varphi+\left(z_{1}-z_{2}\right)\left(\beta_{1} \beta_{2}-\cos \varphi\right)\right. \\
& \left.+\operatorname{ct}\left(\beta_{1} \beta_{2}-1\right) \sin \varphi\right] .
\end{aligned}
$$


TABLE II. Kick parameters for different crossing angle and different low energy beam size for the example situation in Sec. III A.

\begin{tabular}{lccccccccc}
\hline \hline Case & $\varphi\left[^{\circ}\right]$ & $\sigma_{x}[\mu \mathrm{m}]$ & $\sigma_{y}[\mu \mathrm{m}]$ & $\sigma_{z}[\mu \mathrm{m}]$ & $\theta_{y, \max }[\mu \mathrm{rad}]$ & $\theta_{x, \max }[\mu \mathrm{rad}]$ & $\tau_{\mathrm{RMS}}[\mathrm{fs}]$ & $\Delta \varepsilon_{y, \text { kick }}\left[\% \varepsilon_{y}\right]$ & $\Delta \varepsilon_{x, \mathrm{kick}}\left[\% \varepsilon_{x}\right]$ \\
\hline 1 & 45 & 30 & 35 & 35 & 9.92 & 8.7 & 100 & 51.2 & 0.4 \\
2 & 45 & 35 & 30 & 35 & 9.60 & 8.1 & 105 & 50.5 & 0.4 \\
3 & 45 & 35 & 35 & 30 & 9.25 & 8.0 & 106 & 47.6 & 0.4 \\
4 & 45 & 35 & 35 & 35 & 9.00 & 7.9 & 107 & 45.7 & 0.4 \\
5 & 90 & 35 & 30 & 35 & 14.0 & 13.0 & 185 & 183 & 1.0 \\
6 & 90 & 30 & 35 & 35 & 13.5 & 12.9 & 185 & 169 & 1.0 \\
7 & 90 & 30 & 35 & 30 & 14.0 & 13.6 & 179 & 177 & 1.0 \\
8 & 90 & 35 & 35 & 35 & 13.0 & 12.3 & 190 & 163 & 1.0 \\
\hline \hline
\end{tabular}

Following the similar process of deriving the vertical kick $\theta_{y}$, we have the horizontal kick function as follows:

$$
\begin{aligned}
\theta_{x r}= & -\frac{e q_{2} Z_{0} c}{2 \pi E_{1}} \times \frac{(1-\cos \varphi)\left[1+\gamma_{2}^{2} \beta_{1} \beta_{2}+\left(1-\gamma_{2}^{2}\right) \cos \varphi\right]}{\gamma_{2} \sqrt{\gamma_{2}^{2}(1-\cos \varphi)^{2}+\sin ^{2} \varphi}} \\
& \times \frac{1}{\sqrt{2\left[\sigma_{x}^{2} \sin ^{2} \varphi+\sigma_{z}^{2}(1-\cos \varphi)^{2}\right]}} \times f_{x}\left(\rho, \bar{u}_{1}, \bar{y}_{1}\right)
\end{aligned}
$$

with the profile function

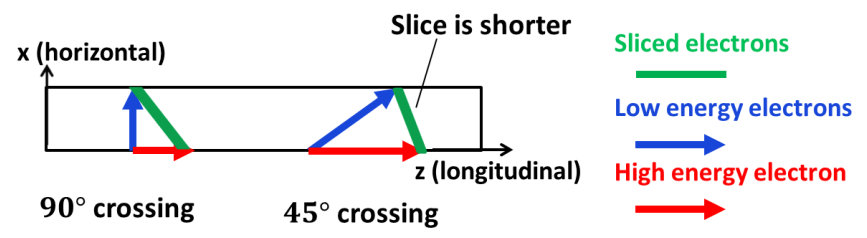

FIG. 10. Illustration of using $45^{\circ}$ crossing to reduce crossing time and reduce the slice pulse length.

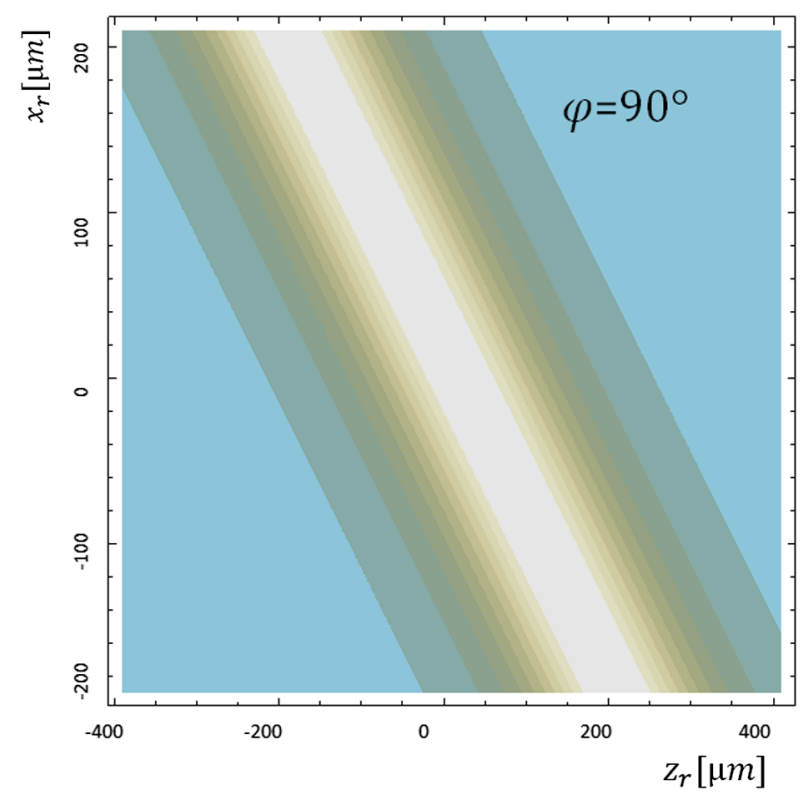

$$
\begin{aligned}
f_{x}\left(\rho, \bar{u}_{1}, \bar{y}_{1}\right)= & \int_{0}^{\infty} \operatorname{Re}\left[W\left(-\bar{y}_{1}+i u\right)\right] \\
& \times\left[e^{-\left(\frac{u}{\rho}+\bar{u}_{1}\right)^{2}}-e^{-\left(\frac{u}{\rho}-\bar{u}_{1}\right)^{2}}\right] d u
\end{aligned}
$$

and the same scaling parameters $\rho, \bar{u}_{1}, \bar{y}_{1}$ as $\theta_{y}$ in Eq. (15). Notice that the horizontal profile function $f_{x}$ is different from the vertical profile function $f_{y}$ in Eq. (14). The plot of the profile function $f_{x}$ in Fig. 13 shows the maximum and minimum kick value located at $\bar{y}_{1}=0, \bar{u}_{1}=-1$ and $\bar{y}_{1}=0, \bar{u}_{1}=1$, respectively. We also plot the profile $f_{x}$ as a function of $\bar{u}_{1}$ on axis $\bar{y}_{1}=0$ in Fig. 14 and as a function of $\bar{y}_{1}$ on axis $\bar{u}_{1}=-1$ in Fig. 15. Figure 14 and Fig. 15 are different from the vertical kick's plots Fig. 4 and Fig. 3 in their dependence with the scaling parameter $\rho$. With the increase of $\rho, f_{x}$ increases, while $f_{y}$ decreases. The increase of the angular divergence increases the emittance of the storage ring bunch and then sets limitation on the system's repetition rate [13]. For the parameters in the example in Sec. III A, the maximum of the horizontal kick angle is

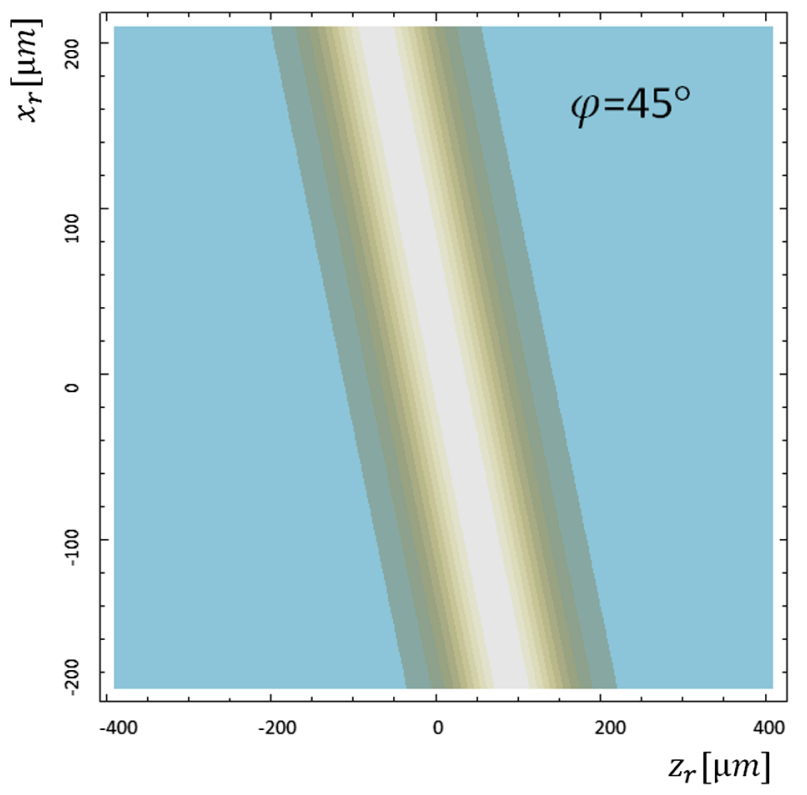

FIG. 11. Contour plots of the kick angle in $x z$ phase space cutting at $y=0$ for different crossing angle $\varphi=90^{\circ}, \varphi=45^{\circ}$. Show the reduction of the crossing angle can reduce the slice width induced by the crossing time. 


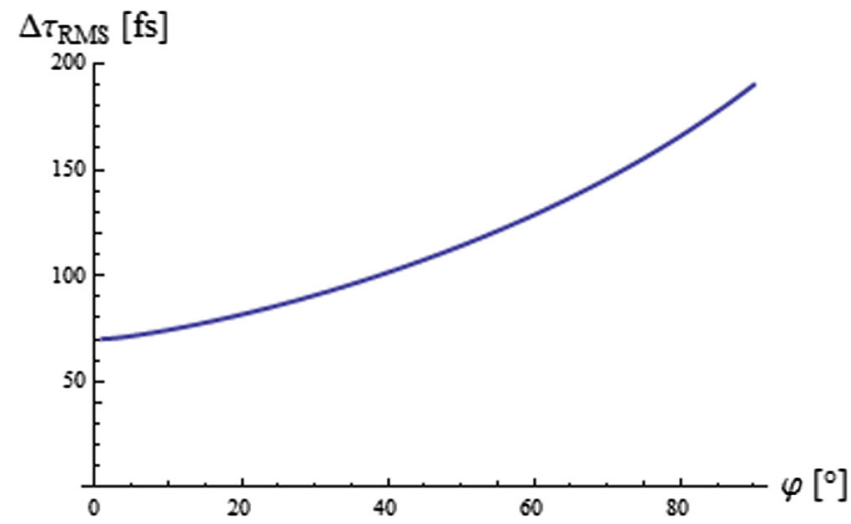

FIG. 12. Estimated slice bunch width $\tau_{\text {RMS }}$ is reduced with the decrease of the crossing angle $\varphi$.

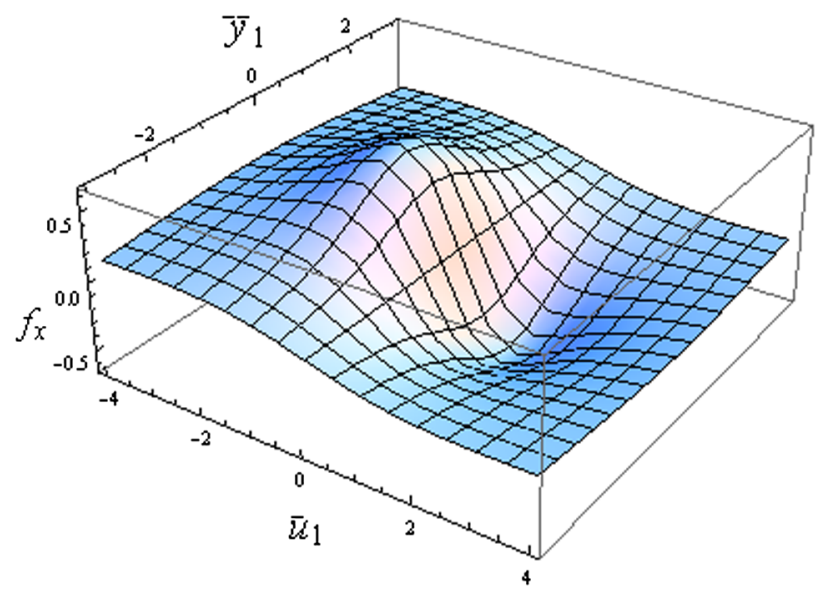

FIG. 13. Profile function $f_{x}$.

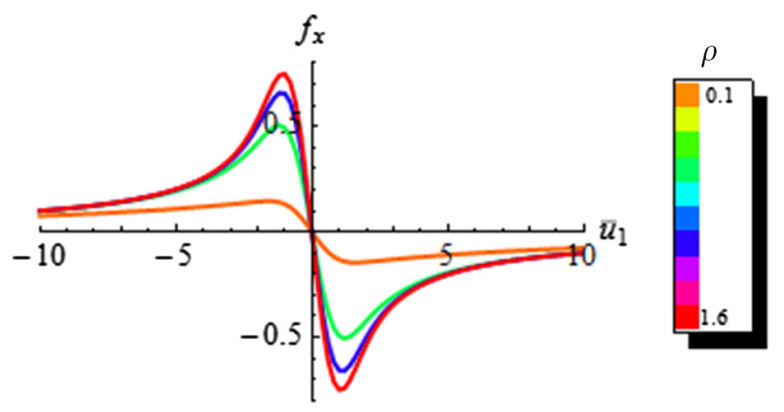

FIG. 14. Profile on axis $\bar{y}_{1}=0$. The maximum $f_{x}$ locates at $\bar{u}_{1}=-1$. Large $\rho$ corresponds to large $f_{x}$.

$12 \mu \mathrm{rad}$ for $90^{\circ}$ crossing and $7.9 \mu \mathrm{rad}$ for $45^{\circ}$ crossing. The estimated horizontal emittance increase due to the horizontal divergence increase is $1 \% \epsilon_{x}$ for $90^{\circ}$ and $0.4 \% \epsilon_{x}$ for $45^{\circ}$, respectively. The horizontal emittance increase is much smaller than the vertical emittance increase due to the vertical divergence increase which is $163 \% \epsilon_{y}$ for $90^{\circ}$ and $45.7 \% \epsilon_{y}$ for $45^{\circ}$, respectively. More numerical examples are given in Table II. Therefore the repetition rate
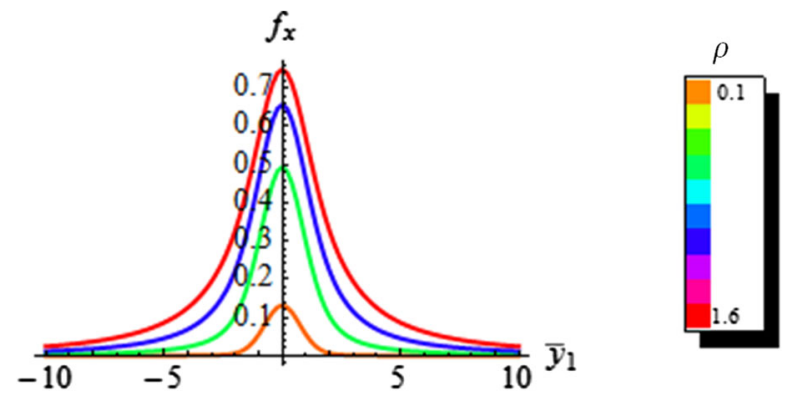

FIG. 15. Profile on axis $\bar{u}_{1}=-1$. Large $\rho$ corresponds to large $f_{x}$.

of the e-beam slicing system is limited by the vertical emittance increase and the horizontal angular divergence increase can be ignored.

\section{B. Longitudinal energy modulation $\Delta \gamma$}

According to Eq. (4), the longitudinal force exerting on the storage ring bunch by the low energy bunch can be expressed as

$$
\begin{aligned}
F_{z r}= & \frac{e q_{2}}{4 \pi \varepsilon_{0}} \frac{\gamma_{2}}{S^{3}} \times\left[\left(x_{1}-x_{2}\right) \cos \varphi\right. \\
& \left.+\left(z_{1}-z_{2}\right) \sin \varphi+\operatorname{ct}(1-\cos \varphi)\right] .
\end{aligned}
$$

The energy change induced by the longitudinal force $F_{z r}$ is

$$
d \gamma_{1}=\frac{F_{z r}}{m c^{2}} d z_{r}=\frac{F_{z r}}{m c} d t
$$

We integrate $d \gamma_{1}$ over the crossing time to obtain the energy modulation $\Delta \gamma_{\text {point charge }}$ induced by a point charge in the low energy bunch 2 . Then we integrate $\Delta \gamma_{\text {point charge }}$ over the whole low energy bunch 2 . Finally, we obtain the slice energy modulation induced by the low energy bunch's longitudinal force exerted on the storage ring bunch as follows:

$$
\begin{aligned}
\Delta \gamma= & -\frac{e q_{2} Z_{0} c}{2 \pi m c^{2}} \times \frac{\left[\gamma_{2}^{2}(1-\cos \varphi)+\cos \varphi\right] \sin \varphi}{\gamma_{2} \sqrt{\gamma_{2}^{2}(1-\cos \varphi)^{2}+\sin ^{2} \varphi}} \\
& \times \frac{1}{\sqrt{2\left[\sigma_{x}^{2} \sin ^{2} \varphi+\sigma_{z}^{2}(1-\cos \varphi)^{2}\right]}} \times f_{x}\left(\rho, \bar{u}_{1}, \bar{y}_{1}\right)
\end{aligned}
$$

where $f_{x}\left(\rho, \bar{u}_{1}, \bar{y}_{1}\right)$ is given by Eq. (20). For the beam parameters in the example in Sec. III A, the maximum energy modulation is $\Delta \gamma=0.072$ at $90^{\circ}$ crossing and $\Delta \gamma=0.11$ at $45^{\circ}$ crossing. Therefore compared with $\gamma=6000(3 \mathrm{GeV})$, the energy modulation induced by the Coulomb force can be ignored. 


\section{REACTION OF STORAGE RING BUNCH TO LINAC BUNCH}

To calculate the reaction of high energy bunch to low energy bunch, we use Eq. (13) and change notation. Now "2" refers to high energy bunch and " 1 " refers to low energy bunch. Then for the high energy bunch, the axis " $+\mathrm{x}$," " $+\mathrm{y}$ " and " $\mathrm{z}$ " are the longitudinal, vertical, and horizontal direction, respectively. Here, we use the beam parameters in Sec. III A as an example to explain that the reaction can be ignored.

At the kick point, the relevant parameters of the high energy bunch are $\sigma_{x}=15 \mathrm{ps} \times 3 \times 10^{8} \mathrm{~m} / \mathrm{s}=4.5 \mathrm{~mm}$, $\sigma_{y}=\sqrt{25 \mathrm{~m} \times 10^{-11} \mathrm{~m}}=15.8 \mu \mathrm{m}, \sigma_{z}=\sqrt{3.8 \mathrm{~m} \times 10^{-9} \mathrm{~m}}=$ $61.6 \mu \mathrm{m}, q_{2}=\frac{0.5 \mathrm{~A} \times 2.63 \mu \mathrm{s}}{1000}=1.3 \mathrm{nC}$ (For beam current of $0.5 \mathrm{~A}$, assume 1000 bunches, with the revolution time of $2.63 \mu \mathrm{s}$, we have the bunch charge as $1.3 \mathrm{nC}$ ), $E_{1}=20 \mathrm{MeV}, \gamma_{2}=\frac{3 \mathrm{GeV}}{0.511 \mathrm{MeV}}=6000$. In order to estimate the maximum reaction, we choose crossing angle $\varphi=90^{\circ}$, then $\theta_{y}\left(90^{\circ}\right)=\frac{e q_{2} Z_{0} c}{2 \pi E_{1}} \frac{\gamma_{2}}{\sqrt{\gamma_{2}^{2}+1}} \frac{1}{\sqrt{2} \sigma_{y}} \times f_{y}\left(\rho, \bar{u}_{1}, \bar{y}_{1}\right)=0.05 \times$ $f_{y}\left(\rho, \bar{u}_{1}, \bar{y}_{1}\right)$, where $f_{y}\left(\rho, \bar{u}_{1}, \bar{y}_{1}\right)$ is the profile function. For this storage ring bunch, the profile parameter $\rho=\sqrt{\frac{\gamma_{2}^{2}}{\gamma_{2}^{2}+1} \cdot \frac{\sigma_{x}^{2}+\sigma_{z}^{2}}{\sigma_{y}^{2}}}=285$. We choose the same situation as Sec. III A, then $\bar{u}_{1}=0, \bar{y}_{1}=1$. When $\rho \gg 1$, the profile function Eq. (14) becomes

$$
\begin{aligned}
f_{y} & \left(\rho \gg 1,0, \bar{y}_{1}=1\right) \\
= & \int_{0}^{\infty} \operatorname{Re}[W(i y)]\left[e^{-\left(\rho y-\bar{y}_{1}\right)^{2}}-e^{-\left(\rho y+\bar{y}_{1}\right)^{2}}\right] d y \\
= & \frac{1}{\rho} \int_{0}^{\infty} \operatorname{Re}\left[W\left(i \frac{y}{\rho}\right)\right]\left[e^{-\left(y-\bar{y}_{1}\right)^{2}}-e^{-\left(y+\bar{y}_{1}\right)^{2}}\right] d y .
\end{aligned}
$$

Due to $\bar{y}_{1}=1$, the contribution of the factor $\left[e^{-\left(y-\bar{y}_{1}\right)^{2}}-\right.$ $e^{\left.-\left(y+\bar{y}_{1}\right)^{2}\right]}$ to the profile function $f_{y}$ comes from $y \sim 1$, then we have $y / \rho \ll 1$. This leads to $W\left(i \frac{y}{\rho}\right)=1$. Then we have

$$
\begin{aligned}
f_{y}\left(\rho \gg 1,0, \bar{y}_{1}=1\right) & \approx \frac{1}{\rho} \int_{0}^{\infty}\left[e^{-\left(y-\bar{y}_{1}\right)^{2}}-e^{-\left(y+\bar{y}_{1}\right)^{2}}\right] d y \\
& =\frac{\sqrt{\pi}}{\rho} \operatorname{erf}\left(\bar{y}_{1}\right),
\end{aligned}
$$

where $\operatorname{erf}(z)=\frac{2}{\sqrt{\pi}} \int_{0}^{z} e^{-t^{2}} d t$. Therefore, the profile function $f_{y}\left(\rho=285, \bar{u}_{1}=0, \bar{y}_{1}=1\right) \approx 0.0052$ and the reaction angle on the linac bunch from the storage ring bunch is $\theta_{y}\left(90^{\circ}\right)=0.087 \times f_{y}\left(\rho=285, \bar{u}_{1}=0, \bar{y}_{1}=1\right) \approx 0.26[\mu \mathrm{rad}]$. We assume the longitudinal beam size of the linac bunch is $35 \mu \mathrm{m}$. Then during the interaction time, the linac bunch's position will be changed about $0.016 \mu \mathrm{m}$ in vertical direction which can be ignored when compared with the vertical beam size $35 \mu \mathrm{m}$.

\section{CONCLUSIONS}

We presented formulas in Eq. (13), Eq. (19), and Eq. (23), which can be used to study the characteristics of the electron beam slicing. The slice bunch's profile is described by the profile function $f_{y}, f_{x}$. The maximum kick angle and the slice width can be manipulated through the low energy beam size $\sigma_{x}, \sigma_{y}, \sigma_{z}$ and the crossing angle $\varphi$. With the variance of the crossing angle $\varphi$, the relative importance of the low energy beam size $\sigma_{x}, \sigma_{y}, \sigma_{z}$ will be varied. Decreasing the crossing angle $\varphi$ can significantly shorten the slice width at the expense of lowering the maximum kick angle $\theta_{y \text {,max }}$. Because the slice width is independent of the charge of the low energy bunch, the decrease of $\theta_{y, \max }$ induced by the reduction of $\varphi$ can be compensated by increasing the charge and the energy of the low energy bunch while keeping the same 3-D beam size. The influence of the horizontal angular divergence increase of the slice can be ignored, because the limitation of the system's repetition rate is set by the vertical emittance increase. The energy modulation of the slice induced by the kick is very small and can be also ignored. The reaction of the storage ring bunch on the low energy linac bunch can be ignored due to the small position change of the linac bunch included by the reaction. The formulas and the results present here are applied to the study of electron beam slicing in NSLS-II [13] and also are used to determine the target parameters of the low energy bunch to guide the design of the linac compressor [15].

\section{ACKNOWLEDGMENTS}

We would like to thank all colleagues of the accelerator group in NSLS-II BNL. This work was funded by DOE under Contracts No. LDRD12-023 and LDRD14-022.

[1] S. Ozaki, J. Bengtsson, S. Kramer, S. Krinsky, and V. Litvinenko, in Proceedings of the 22nd Particle Accelerator Conference, PAC-2007, Albuquerque, NM (IEEE, New York, 2007), p. 77.

[2] A. Zholents and M. Zolotorev, Phys. Rev. Lett. 76, 912 (1996).

[3] R. W. Schoenlain, S. Chattopadhyay, H. H. W. Chong, T. E. Glover, P. A. Heimann, C. V. Shank, A. A. Zholents, and M. S. Zolotorev, Science 287, 2237 (2000).

[4] K. Holldack, T. Kachel, S. Khan, R. Mitzner, and T. Quast, Phys. Rev. ST Accel. Beams 8, 040704 (2005)

[5] G. Ingold, A. Streun, B. Singh, R. Abela, P. Beaud, G. Knopp, L. Rivkin, V. Schlott, Th. Schmidt et al., in Proceedings of the Particle Accelerator Conference, Chicago, IL, 2001 (IEEE, New York, 2001), p. 2656.

[6] C. Steier et al., in Proceedings of the 2003 Particle Accelerator Conference, Portland, OR (IEEE, New York, 2003), p. 397. 
[7] O. Chubar and P. Elleaume, in Proceedings of the 6th European Particle Accelerator Conference, Stockholm, 1998 (IOP, London, 1998), p. 1177.

[8] A. Zholents, P. Heimann, M. Zolotorev, and J. Byrd, Nucl. Instrum. Methods Phys. Res., Sect. A 425, 385 (1999).

[9] M. Katoh, Jpn. J. Appl. Phys. 38, L547 (1999).

[10] M. Borland, Phys. Rev. ST Accel. Beams 8, 074001 (2005).

[11] P. Emma, Nat. Photonics 4, 641 (2010).

[12] F. Willeke and L.H. Yu, in Proceedings of the 4th International Particle Accelerator Conference,
IPAC-2013, Shanghai, China, 2013 (JACoW, Shanghai, China, 2013).

[13] A. He, F. Willeke, and L. H. Yu, Phys. Rev. ST Accel. Beams 17, 040701 (2014).

[14] L. D. Landau and E. M. Lifshitz, The Classical Theory of Fields (Addison-Wesley Press, New York, 1951), p. 99.

[15] A. He et al., in Proceedings of the 4th International Particle Accelerator Conference, IPAC-2013, Shanghai, China, 2013 (JACoW, Shanghai, China, 2013). 